\title{
Percepción de las dimensiones de Responsabilidad Social Universitaria (RSU) de parte del grupo de interés interno de la Universidad de Costa Rica (UCR)
}

\author{
Perception of the dimensions of University Social Responsibility (USR) by the \\ internal stakeholders of the University of Costa Rica (UCR)
}

Fabián Herrera Céspedes

Universidad Estatal a Distancia,

Costa Rica

fabian_herrera25@hotmail.com

\section{RESUMEN:}

Este artículo promueve un acercamiento a lo que se entiende como responsabilidad social universitaria, mediante un análisis del concepto propiamente dicho y de la percepción que manifiesten los sectores de interés internos (administrativos, estudiantes y docentes) de la Universidad de Costa Rica. Además, se ofrece una visión conglomerada de las dimensiones personales, universitarias y sociales de este concepto, no solo determinando si una institución denominada socialmente responsable cumple con lo que se espera de ella, sino estableciendo un contraste entre las acciones que la institución ejecuta y lo que realmente implementa en su quehacer cotidiano. Por último, se presenta una serie de resultados relevantes que desembocan en la emisión de conclusiones y recomendaciones de cara a la propuesta de un modelo que pueda ser aplicado para fortalecer a las universidades.

\section{ABSTRACT:}

This article promotes a comprehensive approach to what is understood as university social responsibility through an analysis of the concept per se and of the perception expressed by the internal stakeholders of the University of Costa Rica, such as the administrative personnel, students and educators. Moreover, this research provides a joined vision of the personal, university and social dimensions of this concept, not only by determining whether an institution called socially responsible complies with what is expected, but also by establishing a contrast between the actions that the institution executes and what it really implements in its daily work. Finally, the paper presents a series of relevant results leading to conclusions and recommendations allowing the proposal of a model that may be implemented so as to strengthen higher education institutions.

\section{PALABRAS CLAVES}

RESPONSABILIDAD

SOCIAL,

RESPONSABILIDAD

SOCIAL UNIVERSITARIA,

GRUPOS INTERESADOS
KEYWORDS

SOCIAL

RESPONSIBILITY

UNIVERSITY SOCIAL

RESPONSIBILITY,

STAKEHOLDERS.

\section{RESUMO:}

Este artigo promove uma abordagem do que é entendido como responsabilidade social universitária, por meio de uma análise do próprio conceito e da percepção que manifestem os setores de interesse interno (administrativos, estudantes e docentes) da Universidade de Costa Rica. Além disso, é oferecida uma visão conjunta glomerada das dimensões pessoais, universitárias e sociais desse conceito, não apenas determinando se uma instituição chamada socialmente responsável atende ao esperado dela, mas estabelecendo um contraste entre as ações que a instituição executa e o que realmente implementa no seu trabalho diário. Por fim, é apresentada uma série de resultados relevantes que resultam na emissão de conclusões e recomendações frente à proposta de um modelo que possa ser aplicado para fortalecer as universidades.

\section{RÉSUMÉ:}

Cet article favorise une approche de ce qui est entendu comme responsabilité sociale universitaire au moyen d'une analyse du concept en soi et de la perception que manifestent les secteurs d'intérêt internes (personnel administratif, étudiants et enseignants) de l'Université du Costa Rica. En outre, cet article offre une vision convergente des dimensions personnelles, universitaires et sociales de ce concept, non seulement en déterminant si une institution appelée socialement responsable répond à ce qui est attendu d'elle, mais aussi en établissant un contraste entre les actions que l'institution exécute et ce qu'elle met réellement en pratique dans ses activités journalières. Finalement, une série de résultats importants est présentée qui débouchent sur des conclusions et recommandations en vue de proposer un modèle qui puisse être appliqué pour consolider les universités. 


\section{INTRODUCCIÓN}

En la actualidad, establecer relaciones dinámicas y proactivas entre los elementos de la hélice quíntuple de la innovación (Gobierno, universidad, empresa + comunidad y ambiente) se ha convertido en un factor preponderante para demostrar el rol social, transformador y generador que tienen las instituciones de educación superior, especialmente aquellas de orden público. Esto es ocasionado porque hoy las labores cotidianas de las universidades transcienden las funciones que se concebían como tradicionales y que, se supone, se encuentra insertas dentro de lo que ellas mismas realizan en los ámbitos de la acción social, la investigación o la docencia. Además, esta situación puede influenciar profundamente los procesos de gestión y redefinir los sistemas aplicados en cada región para desarrollar las áreas sociales, económicas, industriales y culturales.

Las universidades, sin importar que el capital social sea de orden privado o público, deben garantizar el libre acceso y gratuidad de los conocimientos que generan, procurando de esta forma modificar a la sociedad en sus niveles más profundos. En cuanto a estos cambios, deben rescatarse algunos aspectos relevantes y que deben ser afrontados, ya que generan expectativas dentro de las organizaciones, entre las cuales podemos encontrar las siguientes: 1 . la propia sobrevivencia de la organización se ve afectada por el cambio en el entorno; 2. el cambio por sí mismo genera oportunidades nuevas para aprovechar y progresar; 3. la propia estructura y los elementos particulares organizacionales afectan directamente el cambio en cuanto a su tiempo de aplicación.

Producto de las demandas de adaptación ocasionadas por la globalización, específicamente en cuanto a la necesidad de respuestas prontas y efectivas a dificultades actuales, las empresas privadas y las instituciones públicas se ven obligadas a tomar el control de sus procesos y reconceptualizar sus modelos de responsabilidad social; es decir, tomar conciencia sobre la forma en que sus acciones afectan a terceros. Bajo esa línea de ideas, es posible evidenciar que hoy el instaurar modelos de responsabilidad social (RS) se ha convertido en tendencia creciente o, en otras palabras, en una moda, la cual pone en manifiesto las obligaciones que las empresas e industrias tienen con la sociedad.

En el año 2004, en Suecia, enmarcada en la reunión del Consejo ISO, se llevó a cabo la Conferencia Mundial de Responsabilidad Social, donde se postulan los reglamentos y normas de estandarización aplicables a la RS. Esta regulación se desarrolla mediante el establecimiento de esquemas por áreas de distintos sectores: ambiente laboral, comunal, social y ético, elementos que a la postre concretan lo que hoy conocemos como normas internacionales de calidad (ISO). Bajo esta perspectiva, es importante rescatar que, dentro del sector académico, son las universidades las promotoras de iniciativas, propuestas, procesos de innovación y alternativas a las demandas sociales, y las cuales requieren un análisis profundo y permanente.

Específicamente, en lo que respecta a la responsabilidad social universitaria (RSU), el origen de esta teoría corresponde al eje fundamental de brindar respuestas alternativas a la problemática del sector académico, con sustento teórico y documentado. Ahora bien, es imprescindible comprender que la academia no solo atiende a lo que le demande la sociedad, sino que, en conjunto con esta labor medular, corresponde a las universidades gestar propuestas de desarrollo y cambio social, resguardar los principios éticos y morales, procurar el respeto a la libertad en cualquiera de sus manifestaciones, promover los procesos de tolerancia; pero, ante todo, brindar soluciones prontas y efectivas a los conflictos de la sociedad actual, tal como se les demanda.

Con todo lo anterior presente, una institución que acate los elementos y los principios que le determinen como socialmente responsable, debe procurar la transferencia del conocimiento y la dotación a la sociedad de soluciones pertinentes, sin distinción del origen o abordaje de lo que se entiende por responsabilidad social; transformando a la universidad en un instrumento generador y difusor del conocimiento. Estas instituciones también deben expresar panoramas claros de labores y procurar la transparencia mediante la rendición de cuentas y un sistema efectivo de comunicación, tanto con sus sectores interesados como con los externos, nutridos mediante sociedades de conocimiento y transferencia tecnológica, elementos que establecen coherencia entre lo que verdaderamente realizan y lo que la sociedad demanda, es decir, pasar del discurso a la acción. 
En este sentido, es importante rescatar que toda universidad debe cumplir indiscutiblemente, como función, con la proyección social con los grupos externos a ella, mediante los pilares que plantea la educación superior (investigación, acción social, docencia y vida estudiantil), motivo por el cual deben instaurar actividades que pongan en práctica las diferentes experiencias y conocimientos que han sido generadas en torno a la academia; en este mismo sentido, la universidad debe permitir y adaptar lo particular y específico de la sociedad, así como respetar las individualidades, potencialidades y demandas de los sectores, estableciendo los mecanismos idóneos para la interrelación entre todos los participantes.

Por estas razones, y producto de una investigación realizada en la Universidad de Costa Rica, este artículo analizará los estados de percepción sobre RSU manifiestos dentro de los grupos de interés, en específico los correspondientes al sector interno de esta institución (administrativos, docentes y estudiantes), mediante el desarrollo de un plan de trabajo mixto, el cual incorporó elementos de análisis tanto de orden cuantitativo como cualitativo, que alimentaron el estudio y que brindaron una explicación posible al problema de investigación, el cual conjuntamente con las propias demandas globales del sector de la educación superior, potenciaron la investigación para satisfacer el vacío de conocimiento experimentado, mediante un análisis de situación el cual por último permitió brindar una serie de conclusiones y recomendaciones sobre la responsabilidad social universitaria.

Al desarrollar este estudio, se profundizará en los elementos introductorios, sección que presentará algunos aspectos teóricos básicos sobre RSU y su aplicación dentro de las casas de enseñanza superior. Consecuentemente, se hará una breve revisión del soporte brindado mediante la metodología que sustenta este análisis y el cual contempla algunas de las posibles teorías aplicables al caso. Como tercer punto, se abordarán brevemente, dentro de los antecedentes, los resultados que, a criterio del investigador, guardan mayor relevancia. En la etapa de desarrollo, se hará una exposición de sugerencias. Para finalizar, se presentará una serie de conclusiones, para pasar luego a una propuesta de recomendaciones que puedan ser de utilidad a la Universidad de Costa Rica.

\section{MARCO TEÓRICO}

\section{Concepto de responsabilidad social universitaria (RSU)}

Determinar el inicio conceptual de lo que se entiende por responsabilidad social universitaria (RSU) es complicado, pues no existe claridad en cuanto a ello. Lo que sí es posible detallar es el proceso de instrumentalización de las casas de enseñanza superior para ajustar las propuestas que hace la responsabilidad social empresarial dentro de los procesos de ventas y servicios. Esta situación es comentada por Gaete (2012), quien señala lo siguiente:

la propia responsabilidad queda sometida al criterio de la utilidad y esta es una medida a la que es difícil ponerle un límite preciso en una institución universitaria $[\ldots]$ entendida en este caso en términos estrictamente económicos, donde lo útil se identifica con lo económicamente rentable (De la Cruz y Sasia, 2008, citados por Gaete, 2012, p.131).

Con estas declaraciones, los autores plasman sin lugar a duda su posición sobre lo que para ellos representa la RSU y la relación que debe desarrollarse entre la academia y los sectores que tienen algún tipo de afectación con las acciones o decisiones tomadas por ella (grupos de interés), especialmente en cuanto a la búsqueda por satisfacer las demandas hechas por la sociedad.

En este sentido, es factible identificar el tono que se le puede asignar a la RSU en lo que respecta a la forma y en cómo hace uso de su presupuesto; en este sentido, Bouer (2012) rescata lo indicado por Bowen (1953) en cuanto a que las universidades no deben prestar particular interés únicamente en lo que se refiere a los ingresos y los gastos, sino 
que adicionalmente deben instaurar dos elementos primordiales: elementos técnicos, los cuales se pueden definir como la acción de proveer a la sociedad de profesionales competentes y capacitados altamente, y elementos morales, los cuales corresponden específicamente a la formación de profesionales con sensibilidad social y que manifiesten habilidades culturales, que puedan ser líderes gremiales, sectoriales y comunales.

Además, resguardando los fines y propósitos que tutelan a las universidades, estas cuentan con las estructuras administrativas que desarrollan los procedimientos que mejor se adapten y que procuren dotar a la institución con el personal idóneo que requiera, así como también los mecanismos que les permitan determinar la admisibilidad estudiantil y sus planes de estudio.

Al respecto, Víctor Martín (2012) señala algunos elementos que permiten identificar a una universidad como entidad socialmente responsable:

- Es una entidad reflexiva para cuidar la vida en sociedad, con miras al futuro, capaz de actuar de acuerdo a sus valores e impulsarlos dentro de los actores sociales. Se autoanaliza en cuanto al grado de cumplimiento de sus proyectos, enmarcados en la ética y valores institucionales, se revisa a sí misma de modo consciente y respetuoso, pero al mismo con efectividad, voluntad y disposición al cambio.

- Es sostenible, realiza su proyecto de modo excelente, con calidad interna y sin afectar el entorno.

- Es sustentable, es excelente, diseña y contribuye al mejoramiento de la sociedad, de la convivencia, del diálogo y del entendimiento.

- Es trascendente, piensa en el sentido del proyecto humano total del cual forma parte, tomando en cuenta a las generaciones futuras.

- Es éticamente inteligente, une todas las inteligencias con miras a proyectos valiosos para la sociedad.

Debe rescatarse que, al conglomerar las conceptualizaciones anteriores, es posible ir instaurando un concepto propio de RSU y que también se van mostrando apartados en común que fortalecen la definición construida, de las cual es posible señalar los siguientes elementos: compromiso, valor y ética profesional, formación académica, responsabilidad profesional y transparencia, entre otros. Dentro de la instauración de una definición teórica del término de RSU, podemos encontrar lo que señalan Vallaeys, De la Cruz y Sasia (2009), quienes indican que, para que las universidades se mantengan en continua y permanente mejora, necesitan cuatro pilares fundamentales:

1. Gestión ética y respeto ambiental.

2. Formar a la sociedad en cuanto a principios y valores que repercutan en su vida en comunidad.

3. Desarrollar y transferir a la sociedad iniciativas que la beneficien y colaboren en su desarrollo.

4. Construir las políticas y estrategias institucionales, tomando en cuenta la opinión de la sociedad en general, especialmente en los temas que impliquen desarrollo sustentable, desarrollo humano y política pública.

Así mismo, Vallaeys, De la Cruz y Sasia (2009) comentan la existencia de tres estrategias de mejora que pueden aplicar las universidades en su quehacer ordinario:

1. Conjunción e integración de los sectores de interesados (externos o internos) de la universidad.

2. Interrelación de la investigación, la extensión docente, la docencia, la malla curricular y la resolución de los fenómenos sociales.

3. Diagnóstico propio y transparencia institucional al momento de declarar o asumir responsabilidad sobre sus acciones ante los grupos de interés. 
Continuando con la determinación de lo que es posible comprender y aplicar como RSU, se rescata la definición brindada por Gaete (2012), la cual especifica tres apartados:

a) Consecuencialistas: da énfasis al análisis multifactorial de resultados, poniendo a disposición planes estratégicos que procuren reducir afectaciones a las partes interesadas.

b) Prospección: prioridad institucional en la reconceptualización de igualdad y justicia social, enfatizando los beneficios de la universidad más que los comunales.

c) Contractual: deber de responder a la totalidad de las necesidades sociales que son planteadas por las entidades de educación superior.

Dentro del trabajo realizado por De la Cruz y Sasia (2008, citados por Gaete, 2012), las definiciones antes señaladas permiten el análisis de diferentes aspectos y grados de avance en cuanto a la construcción de los parámetros de lo que se puede entender como un comportamiento apegado a lo socialmente responsable de la instituciones de enseñanza superior, pero también los que se interrelacionan con otros planteamientos, tales como el desarrollo sostenible y planes estratégicos de desarrollo.

En los enfoques que se detallan, es posible notar que las instituciones universitarias son exigidas a cooperar activa y primariamente en la búsqueda de soluciones a las demandas que plantean los sectores sociales y que inexorablemente las obligan a transformar sus planes y modelos estratégicos, buscando el apego a las perspectivas antes mencionadas.

\section{Modelo basado en valores y principios para determinar las dimensiones de responsabilidad social universitaria manifiesta}

En términos generales, es posible indicar que las labores sustantivas de cualquier casa de enseñanza superior se basan en el desarrollo de cinco funciones esenciales: docencia, investigación, acción social, gestión administrativa y vida estudiantil. Como soporte de estos pilares académicos, es posible encontrar valores y principios, los cuales permiten la ejecución práctica del quehacer cotidiano de las gestiones, además de servir como guía de comportamiento y accionar individual e institucional, ambos elementos fundamentales en una universidad que se denomine socialmente responsable.

El modelo de valores y principios que se plantea cuenta con tres planos: a) plano personal, b) plano social y c) plano universitario. Dentro de estos planos, existen valores y principios que los determinan y que se detallan brevemente a continuación:

\section{Plano personal}

Integridad: principio de coherencia, el cual consiste en concordar lo que se dice con lo que se hace; manifestaciones de transparencia, honestidad y rectitud.

Dignidad: considera al ser humano racional, con libertad y con iguales derechos y deberes; indisoluble entre lo físico, psíquico, biológico, espiritual y social; el ser humano tiene voluntad propia, es capaz de pensar y elegir; tiene sentimientos y puede optar por expresarlos o callarlos; es perfectible y con capacidad de aprender. Ahora bien, estas características, aplicadas a la vida universitaria en común, permiten identificar al ser humano como un fin y no como un medio, situación que conlleva obligaciones a la institución de brindar condiciones para que el individuo alcance dichas características.

Libertad: capacidad del ser humano de hacerse responsable de su vida y la forma en que la lleva en sociedad. Además, esta condición brinda a cada individuo la posibilidad de autorrealizarse; este principio se manifiesta en la relación con el quehacer universitario en la forma en que la institución respeta las formas de pensar, la libertad de agrupación y de credo, la libertad de cátedra, las formas de enseñanza, la opinión y normas de expresión en todas sus formas. 


\section{Plano social}

Equidad y bienestar comunal: acumulación de elementos de orden social, económico, cultural, espiritual y material que facilitan a los individuos alcanzar y mejorar sus niveles de calidad de vida. Dentro de la comunidad universitaria, el cumplimiento de estos supuestos da prueba de una distribución equitativa de los recursos entre todas las partes para el desarrollo de sus actividades.

Ambiente y desarrollo sustentable: proceso que permite la perpetuidad de la especie humana con las mejores condiciones posibles, además de permitir el desarrollo de las actividades sin afectar en forma desmedida el ambiente y la diversidad contenida en él. Además, el desarrollo sustentable consiste en la búsqueda permanente de satisfacer demandas y necesidades sin comprometer el futuro de generaciones venideras. Dentro de la vida universitaria, se debe procurar el mejoramiento en las condiciones laborales y de vida comunal, estableciendo relaciones satisfactorias entre todos los sectores que componen el campus y el ambiente en él.

Respeto a la diversidad: capacidad de dar valor e integrar a cualquier persona sin ningún elemento discriminatorio (sexo, raza, edad, religión y condición social, entre otros).

Convivencia social y solidaria: el ser humano tiene y requiere vivir en sociedad; por lo tanto, debe adaptarse y someterse a las directrices que como grupo se establezcan. Dentro de esta convivencia, los individuos, en plena manifestación de su libertad, pueden colaborar en la resolución de problemáticas colectivas, hecho que pone en manifiesto el ejercicio de la solidaridad. El desarrollo de estos principios dentro del entorno universitario permite identificar el rol personal que desarrolla cada individuo dentro del grupo y, por ende, dar el crédito y el valor a cada uno de ellos. También se fomenta la crítica constructiva y el diálogo entre los distintos sectores.

Democracia y participación ciudadana: compromiso personal adquirido mediante el hecho de ser parte de una sociedad y de una comunidad, dentro de la cual se contribuye a su mejora y transformación, haciendo valer, respetando y resguardando los principios fundamentales de la participación ciudadana en una democracia, tales como igualdad, libertad, dignidad humana y respeto a la individualidad, entre muchos otros.

\section{Plano universitario}

Verdad: el compromiso con la verdad guarda relación directa con los elementos éticos utilizados para obtenerla y resguardarla. Además, se debe tener plena consciencia de los límites y los alcances que se obtuvieron, entre estos resguardar la dignidad humana y el cuidado a los animales. No es cierto decir que, en aras de la búsqueda de la verdad, todo está permitido. Comprometerse con la verdad implica el otorgamiento libre y gratuito del conocimiento que se genera en la academia y que es requerido por la sociedad.

Excelencia: indicador de calidad utilizado dentro de las labores humanas y laborales realizadas dentro de una organización. También establece la relación que existe entre la forma eficaz, eficiente y responsable con que se realizan las tareas y la gestión propia de la universidad. La excelencia guarda una correspondencia directa con las estrategias, procedimientos, metas y fines de la institución.

Transdisciplinariedad e interdependencia: dentro de esta dinámica, es posible establecer la interrelación que desarrolla la universidad con los distintos sectores, tanto internos (administrativos, docentes y estudiantes), como externos (sociedad, industria y Gobierno, entre otros). Dentro de esta relación bilateral, es posible establecer la influencia que se produce entre las partes, tanto en demanda de necesidades como en la obligación que tiene la academia de generar y poner a disposición los conocimientos. Esta interdependencia debe responder a la resolución de problemas más complejos, abarcándolos desde la mayor cantidad de perspectivas y áreas del conocimiento e integrando holísticamente los fenómenos y las soluciones. 


\section{METODOLOGÍA}

Para desarrollar la investigación que dio origen a este artículo, se utilizó una metodología basada en los siguientes puntos:

1. Revisión de literatura existente sobre responsabilidad social (SR) y sobre responsabilidad social universitaria (RSU).

2. Revisión del marco normativo de la Universidad de Costa Rica que regule lo correspondiente a RS y RSU.

3. Revisión de antecedentes nacionales e internacionales sobre estudios realizados que guarden relación con RSU.

4. Reprocesamiento y análisis de resultados generados por Herrera (2018) en su trabajo final de graduación para denominado "La responsabilidad social de la Universidad de Costa Rica: Hacia un modelo de gestión estratégica", realizado en la Universidad Estatal a Distancia de Costa Rica.

5. Reprocesamiento y resumen de los principales hallazgos obtenidos Herrera (2018) en su trabajo final de graduación, denominado "La responsabilidad social de la Universidad de Costa Rica: hacia un modelo de gestión estratégica”, realizado en la Universidad Estatal a Distancia de Costa Rica.

Con el fin de evaluar la percepción que tiene el sector docente, administrativo y estudiantil sobre responsabilidad social universitaria, se diseñó una encuesta adaptada para cada sector. Cada una de estas encuestas se incorporó a la aplicación open source de encuestas en línea denominada LimeSurvey. Además, se construyó un indicador para cada una de las once dimensiones del modelo de RSU considerado.

Todos los indicadores se conforman de la suma de seis variables, cuyas opciones de respuesta podían ser o (si la respuesta era negativa) o 1 (si la respuesta era afirmativa). De tal forma, cada indicador varía entre o (en el peor de los casos) y 6 (en el mejor de los casos). Cada posible valor en relación con el nivel de RSU percibido se recodificó de la siguiente manera: $0=$ Nada, $1=$ Muy poco, $2=$ Poco, $3=$ Regular, $4=$ Mucho, $5=$ Excelente.

Asimismo, se obtuvo una puntuación total de RSU con la suma de los 66 ítems.

Los datos se analizaron mediante el software Microsoft Excel y RStudio (específicamente con las librerías Dplyr y Ggplot2).

\section{Encuesta}

Para efectuar la recolección de datos, se utilizó como herramienta una adaptación de la encuesta diseñada por Jiménez de La Jara (2008). Como población, se tomó a las partes interesadas internas de la Universidad de Costa Rica, las cuales corresponden a administrativos, docentes y estudiantes, para los cuales se definió la cantidad de cien entrevistas para cada grupo, es decir, $\mathrm{n}=300$. 


\section{RESULTADOS}

Se obtuvo una muestra de 102 observaciones del personal administrativo, 100 para el personal docente y 102 para el sector estudiantil de la Universidad de Costa Rica, a través de una encuesta en línea mediante LimeSurvey.

Resultados sector administrativo

Del total de 102 observaciones, $49 \%$ corresponden a mujeres y $51 \%$ a hombres; el $28 \%$ tiene condición interina, mientras que $72 \%$ es personal en propiedad.

En relación con el nivel de conocimiento sobre el tema, 61 \% del personal administrativo sabe qué es responsabilidad social, mientras que solo la mitad sabe qué es responsabilidad social universitaria.

En cuanto a la valoración general que tiene el personal administrativo de la Universidad de Costa Rica sobre el concepto de responsabilidad social universitaria, es posible notar que la distribución también es relativamente normal, con un pequeño sesgo hacia evaluaciones altas. El puntaje promedio en el indicador de RSU es 28,5, lo cual es menos de la mitad de la puntuación máxima posible. La desviación estándar es 16,8. La mitad de los casos se ubica por debajo del valor de 25. En relación con la evaluación por dimensiones, la UCR fue evaluada positivamente en el cuidado al ambiente y el respeto de la diversidad. $80 \%$ del personal administrativo percibe un nivel alto de compromiso con el ambiente y $78 \%$ considera que hay un nivel alto de reconocimiento de la diversidad. Esta información se expone con detalle en la figura 1.

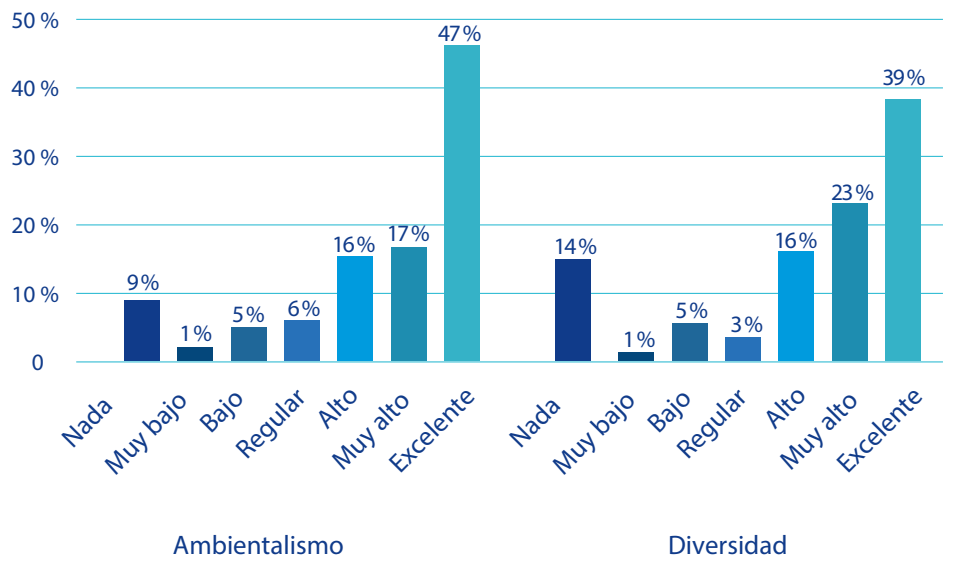

Figura 1. Distribución del nivel de RSU percibido por el personal administrativo en dimensiones con resultados positivos. Fuente: Elaboración propia, 2018. 
Por el contrario, la UCR fue evaluada negativamente por el personal administrativo en los apartados de equidad, democracia, integridad, verdad, interdisciplinariedad y excelencia (de dimensión mejor evaluada a dimensión peor evaluada). $66 \%$ del personal percibe un nivel bajo de equidad e integridad; $67 \%$ un nivel bajo de democracia $y$ verdad; $78 \%$ un nivel bajo de interdisciplinariedad; y, por último, $89 \%$ valora que el nivel de excelencia es bajo. Esta información se presenta en la figura 2.

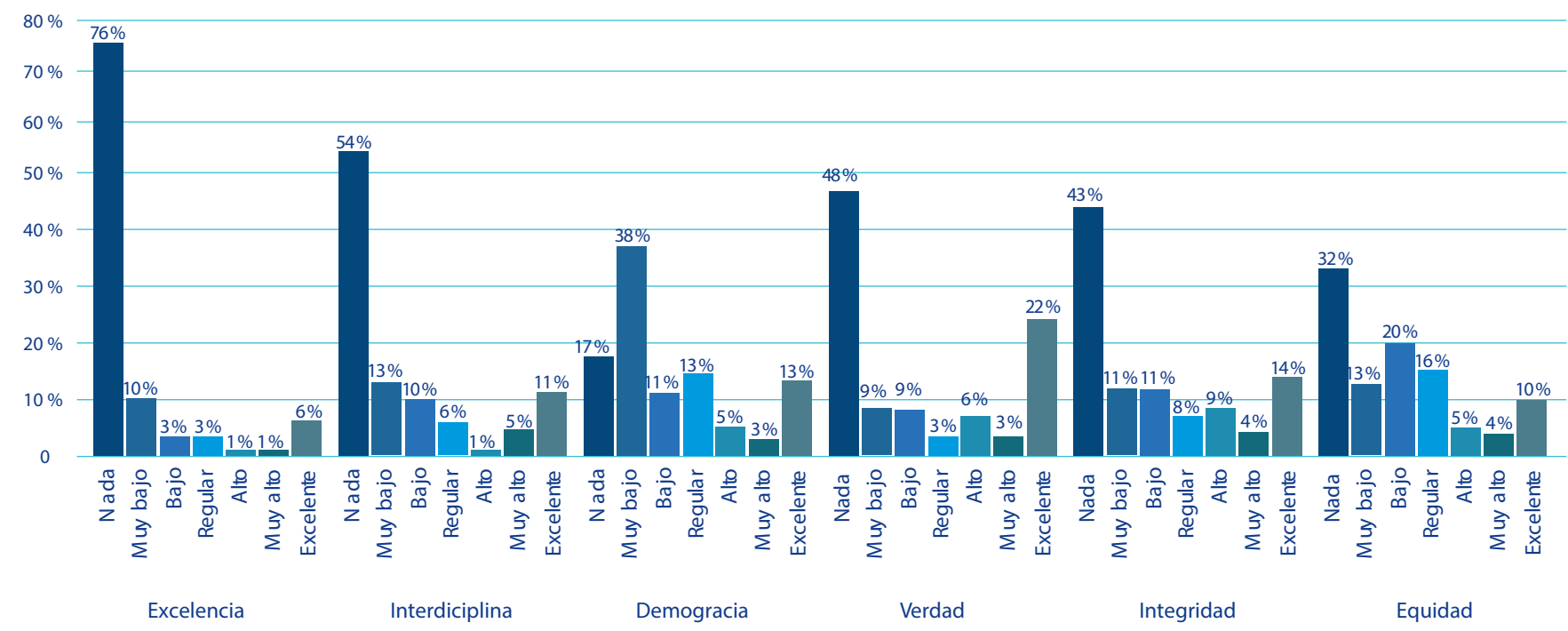

Figura 2. Distribución del nivel de RSU percibido por el personal administrativo en dimensiones con resultados negativos Fuente: Elaboración propia, 2018. 
En relación con el resto de las dimensiones, las distribuciones son menos contundentes, aunque se destacan observaciones relevantes. Por ejemplo, 71 \% del personal valora que el nivel de solidaridad no es alto, $60 \%$ que el nivel de libertad no es alto, $58 \%$ que el nivel de dignidad no es alto.

\section{Resultado sector docente}

Del total de 100 observaciones, $45 \%$ se clasifica como mujer y $55 \%$ como hombre; $37 \%$ de los casos pertenecen a condición interina y el 63 \% a personal con propiedad. La edad media es de 50,1 años con una desviación estándar de 10,5 años. La distribución de esta variable es asimétrica, con una mayor concentración de casos entre los 55 y los 60 años.

Sobre el conocimiento del concepto de responsabilidad social por parte del personal docente, $91 \%$ sabe lo que es. En cuanto al término de responsabilidad social universitaria, 88 \% sabe lo que es. En relación con el puntaje total de RSU, la puntuación media es de 43,2 puntos, con una desviación estándar de 13,3 puntos. La distribución de dichos puntajes está dispersa a lo largo de todo el rango de valores, con dos pequeñas concentraciones en valores bajos y altos. La mediana del puntaje total de RSU es de 43, es decir, la mitad de los casos califican por debajo de este valor y la otra mitad por encima.

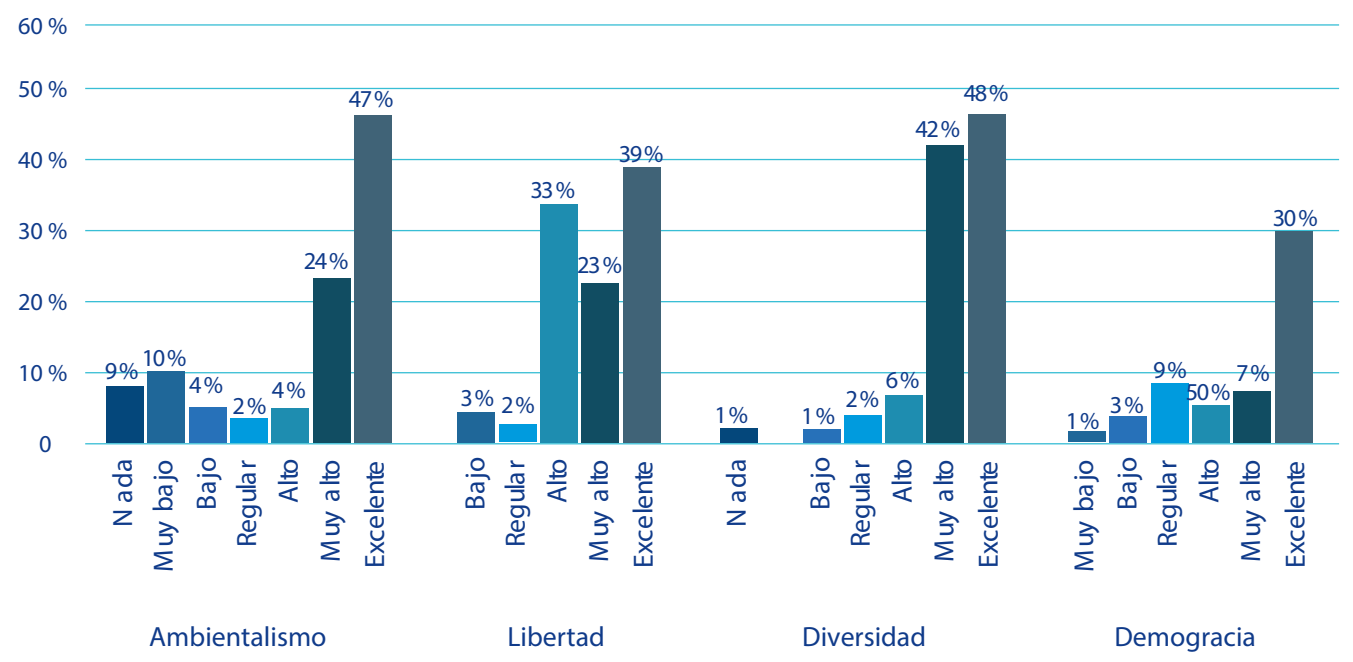

Figura 3. Distribución del nivel de RS percibido por el personal docente según dimensión con resultados positivos. Fuente: Elaboración propia, 2018. 
En cuanto a la valoración por dimensiones, la Universidad de Costa Rica es evaluada de forma positiva por el personal docente en cuatro de once dimensiones. En detalle, 96 \% del personal docente percibe un nivel alto de aceptación de la diversidad, $95 \%$ un nivel alto de libertad, $87 \%$ un nivel alto de democracia y $75 \%$ un nivel alto de ambientalismo. Esta información se aprecia con detalle en la figura 3.

Por otra parte, hay tres dimensiones con evaluaciones negativas. En específico, en orden de la peor evaluada a la mejor evaluada, $48 \%$ del personal docente percibe un nivel de interdisciplina bajo, $46 \%$ un nivel de excelencia bajo y $43 \%$ un nivel de integridad bajo. La figura 4 muestra esta información con mayor detalle.

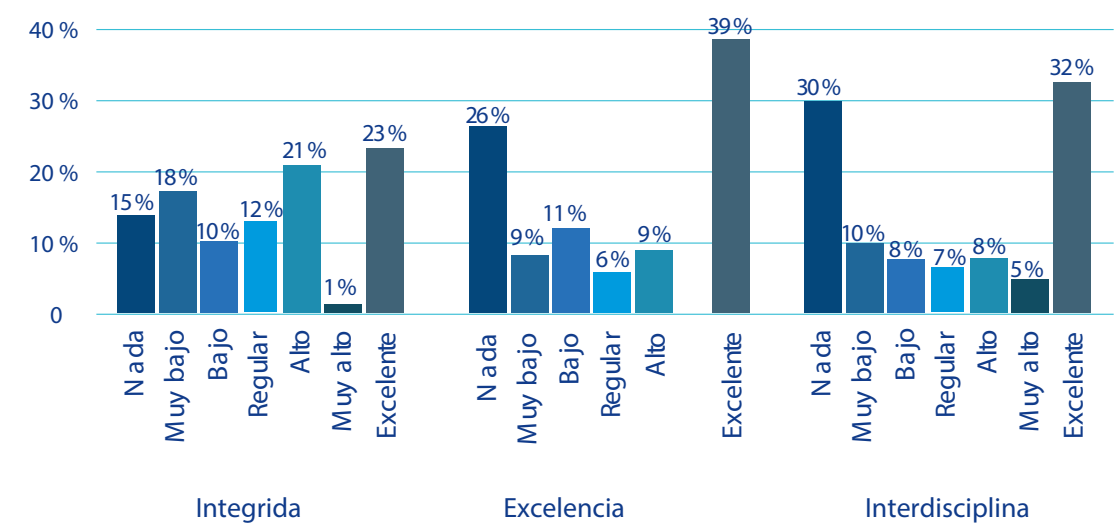

Figura 4. Distribución del nivel de RS percibido por el personal docente en dimensiones con resultados negativos. Fuente: Elaboración propia, 2018. 
En relación con el resto de las dimensiones, se aprecian resultados regulares. Entre algunas observaciones relevantes, se destaca que $53 \%$ del personal docente considera que el nivel de solidaridad no es alto, $52 \%$ que el nivel de verdad no es alto, $51 \%$ que el nivel de equidad no es alto y $45 \%$ que el nivel de dignidad no es alto.

\section{Resultados sector estudiantil}

Sobre el conocimiento de qué es la responsabilidad social por parte del estudiantado, solo $38 \%$ sabe lo que es, mientras que un todavía menor $32 \%$ sabe lo que es responsabilidad social universitaria específicamente.

En relación con el puntaje total de RSU, la puntuación media es de 56,3 puntos con una desviación estándar de 9,3 puntos. La distribución de dichos puntajes es exponencial creciente, con una concentración significativa en valores altos, de tal forma que la mitad de los casos tiene un puntaje total de RSU mayor a 60 puntos. En cuanto a la valoración por dimensiones, la Universidad de Costa Rica es evaluada por el estudiantado de forma positiva en absolutamente todas ellas. En detalle, 99 \% percibe un nivel de aceptación de la diversidad alto, $95 \%$ un nivel de equidad alto, 94 $\%$ un nivel de libertad alto, $93 \%$ un nivel de democracia alto, $92 \%$ un nivel de excelencia alto, $91 \%$ un nivel de interdisciplina alto, $91 \%$ un nivel de solidaridad alto, $87 \%$ un nivel de ambientalismo alto, $81 \%$ un nivel de verdad alto, $78 \%$ un nivel de dignidad alto y, finalmente, $75 \%$ un nivel de integridad alto. Esta información se puede apreciar con detalle en la figura 5 .

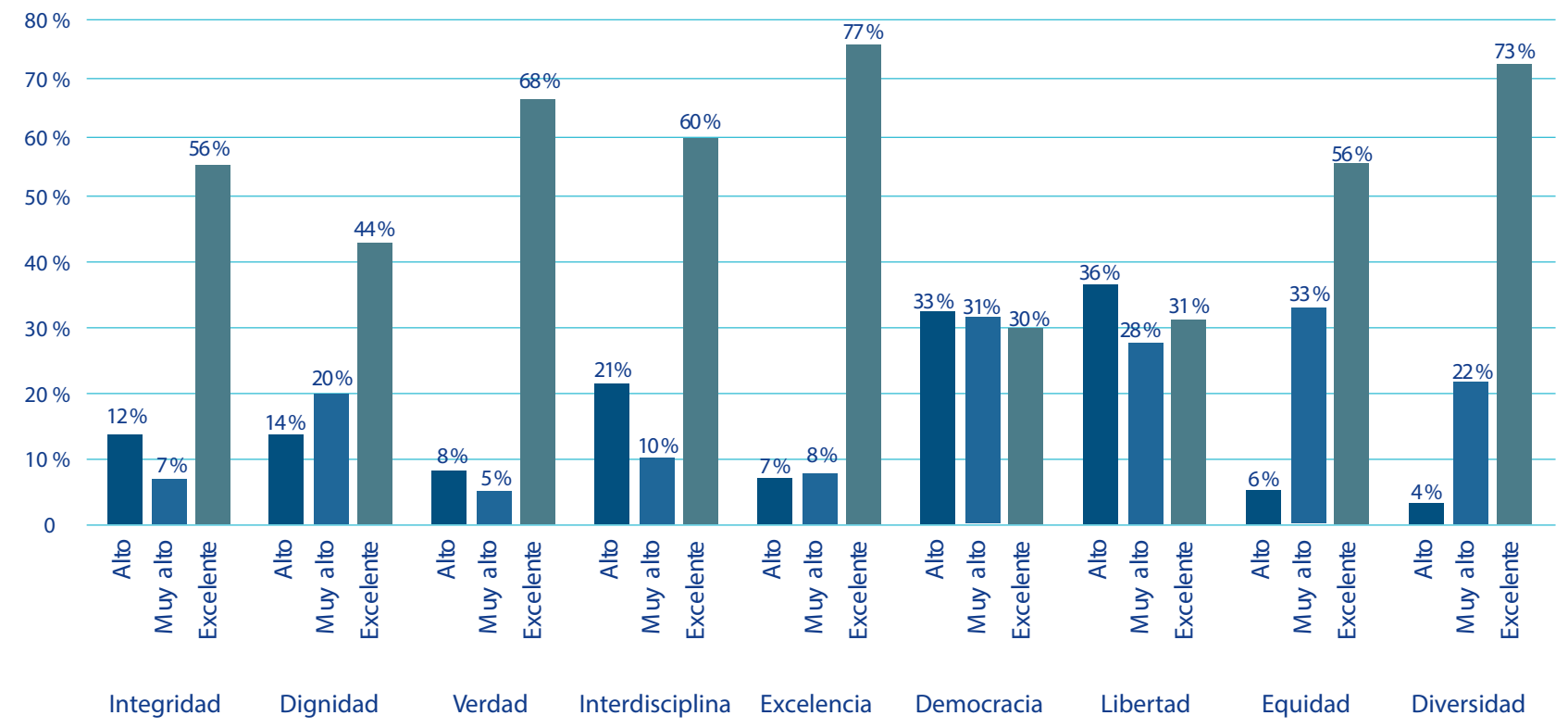

Figura 5. Distribución del nivel de responsabilidad social percibido por el estudiantado en dimensiones con resultados positivos Fuente: Elaboración propia, 2018. 


\section{ANÁLISIS DE RESULTADOS}

1. En el análisis de los datos, es posible identificar algunos de los resultados que manifiestan especial relevancia y los cuales se presentan a continuación:

2. El sector con mayor conocimiento tanto del concepto de RS como de RSU es el sector docente. Con un nivel medio de conocimiento para ambos términos, se encuentra el sector administrativo y el grupo que conoce porcentualmente menos los conceptos es la parte estudiantil.

3. Para el sector administrativo, las dimensiones de la encuesta con resultados positivos fueron la parte ambiental y diversidad social; con resultados medios, es posible identificar los elementos de dignidad, libertad y solidaridad; por último, las dimensiones con resultados negativos corresponden a equidad, democracia, integridad, verdad, interdisciplinariedad y excelencia.

4. Para el sector docente, las dimensiones con puntaje positivo son ambiente y democracia; con resultados medios es posible identificar las áreas de solidaridad, verdad, equidad y dignidad; y en cuanto a dimensiones con percepción negativa, es posible identificar interdisciplinariedad, excelencia e integridad.

5. Dentro del sector estudiantil, con el mayor puntaje de percepción positiva, se encuentran las dimensiones de ambiente, diversidad, equidad, libertad, democracia, excelencia, interdisciplinariedad, solidaridad, ambiente, verdad, dignidad e integridad.

\section{CONCLUSIONES}

La aplicación de modelos de responsabilidad social universitaria desempeña un papel clave dentro de la dinámica nacional e internacional de las instituciones de educación superior, para adaptar y complementar sus labores cotidianas a las necesidades internas y externas de los distintos sectores.

El diseño de estos modelos de responsabilidad social universitaria no solo son una práctica, sino una necesidad que afrontan las universidades para hacer frente a los nuevos requerimientos sociales y económicos, en la búsqueda de satisfacer las necesidades que manifiestan sus partes interesadas (stakeholders).

La Universidad de Costa Rica, al igual que otras universidades públicas o privadas, deben enfrentar el reto no solo de hacer una definición del término de RSU propia y ajustada a sus particularidades, sino de satisfacer una segunda necesidad, la cual consiste en incorporar este concepto dentro de sus políticas internas (plan estratégico y políticas internas, entre otros).

Ahora bien, específicamente dentro de la estructura administrativa de la Universidad de Costa Rica, es posible reconocer iniciativas que diferentes instancias de esta entidad desarrollan y que fomentan la RSU, pero las cuales carecen de articulación grupal y se llevan a cabo individualmente y separadas de la administración central universitaria.

En términos generales, sobre el manejo del concepto de RS y RSU dentro de las distintas partes interesadas de la Universidad de Costa Rica, la encuesta nos permite concluir que el sector que indica tener mayor noción sobre estos elementos corresponde al personal docente. En un nivel intermedio de manejo conceptual se encuentra el sector administrativo y en último puesto se halla el sector estudiantil.

Para los tres sectores (administrativo, estudiantil y docente), las dimensiones de ambiente y diversidad son las que reciben mejor calificación, lo cual evidencia que para la comunidad universitaria el respeto a la diversidad y por ende a las diferencias entre los miembros que la conforman es palpable. Esto nos permite decir que la Universidad de Costa 
Rica es inclusiva. Otro elemento que es importante rescatar es lo que respecta al ambiente, pues, si bien es cierto este apartado es el menos normado por la institución, su puesta en práctica por la población estudiantil es realizada por voluntad propia, es decir, es de orden intrínseco.

Opuesto al punto anterior, los apartados de excelencia, interdisciplinariedad e integridad son calificados de forma negativa mayormente entre los docentes y los administrativos. Esta situación nos permite caracterizar que, para estos grupos de interés, dichos puntos (a los cuales podemos enmarcar dentro de lo que atiende la planificación estratégica y la gestión administrativa), tienen carencias y margen de mejora que los dejan en los niveles inferiores.

Bajo otro punto de análisis, es posible establecer otro tipo de categoría de análisis para los grupos de interés de la UCR (docentes, estudiantes y administrativos), y el cual consiste en clasificar el puntaje que le otorga cada sector a los elementos contenidos dentro del concepto de responsabilidad social universitaria, específicamente, consiste en contabilizar los puntajes de las dimensiones que mayoritariamente caracterizan a una institución de enseñanza superior socialmente responsable, siendo de este modo que el sector estudiantil le confiere a la UCR un alto puntaje al desarrollo de iniciativas institucionales y comunales que sean compatibles concepto de RSU y su dimensión social y ambiental. En esta misma clasificación, se destaca que el sector docente establece con una valoración intermedia, tomando como base el puntaje total de las dimensiones que constituirían a la UCR como una universidad socialmente responsable, rescatando que los ámbitos de integridad ( transparencia y rendición de cuentas), excelencia ( procesos administrativos), y interdisciplinariedad ( contacto y sinergia de labores internas institucionales, se valoran de forma negativa por los docentes.

En último punto del ordenamiento, se distingue al sector administrativo, grupo que no manifiesta satisfacción en casi ninguna de las dimensiones, de no ser por lo ambiental y la diversidad; elementos que, como ya se indicó, son intrínsecos y están estrechamente relacionados con valores personales, principios morales y normas de vida y convivencia en sociedad.

Por último, se observa que el sector estudiantil es el grupo que califica en forma positiva la totalidad de los elementos evaluados dentro de las dimensiones de caracterización de lo que se entiende como universidad socialmente responsable. Esta situación nos permite concluir que las acciones que desarrolla la UCR para satisfacer las necesidades y demandas de los estudiantes han sido efectivas y bien recibidas por dicho sector. Este hecho nos deja claro que las autoridades universitarias y los sectores coadyuvantes (docente y administrativo) tienen plena comprensión de cuál es la razón de ser de la institución y se abocan a cumplir los requerimientos que les plantean; pero, sobre todo, desarrollan labores conjuntas y equilibradas que dan claridad al estudiantado en cuanto a que la UCR es una institución socialmente responsable y orientada en forma plena y favorable hacia ellos.

\section{RECOMENDACIONES}

Desarrollar planes de evaluación y seguimientos anuales que permitan medir los movimientos en los estados de percepción que manifiestan los grupos de interés internos de la UCR y que faciliten el análisis y el proceso de toma de decisiones futuras por parte de las instancias superiores universitarias.

Propiciar espacios de opinión, en los cuales los grupos de interés de la UCR puedan manifestar sus necesidades y requerimientos, además de entablar diálogos constantes en las partes, que sirvan como insumo para elaborar estrategias conjuntas de abordaje.

Diseñar planes de capacitación que permitan el fortalecimiento de los conceptos, alcances y posibilidades que genera la aplicación de modelos de responsabilidad social dentro de los sistemas administrativos, económicos y sociales de las casas de enseñanza superior. 
Divulgar a la comunidad universitaria las acciones, planes y medidas tomadas por la administración, producto de las iniciativas conjuntas y que sean parte de lo que se entiende por RSU.

Diseñar material tanto impreso como digital que facilite la comprensión de las dimensiones que conlleva la determinación de una universidad como socialmente responsable.

Conglomerar las iniciativas individuales que desarrollan las partes interesadas internas de la UCR, en un único modelo de RSU aplicable a la realidad manifiesta de la institución y que sirva de guía para los planes de desarrollo y estratégicos que proponga desarrollar.

\section{REFERENCIAS}

Bowen, H. R. (1953). Social responsibilities of the businessman. Nueva York: Harper-Row. Citado en Bouer, E. (2012). Responsabilidad social de la empresa análisis del concepto. Revista Estudios Económicos, 29(59), 1-30. Recuperado de http://ebour.com.ar/pdfs/Estudios\%2059\%2027\%2011\%202012.pdf

De la Cruz, C. y Sasia, P. (2008). La responsabilidad de la universidad en el proyecto de construcción de una sociedad. Revista Educación Superior y Sociedad, Perú, 13(2),17-52. Citados por Gaete, R. (2012). Responsabilidad social universitaria: una nueva mirada a la relación de la universidad con la sociedad desde la perspectiva de las partes interesadas. Un estudio de caso. (Tesis doctoral). Universidad de Valladolid, Facultad de Educación y Trabajo Social. Valladolid, España. Recuperado de http://uvadoc.uva.es/bitstream/10324/923/1/TESIS148- 120417.pdf

Herrera, F. (2018). La responsabilidad social de la Universidad de Costa Rica: Hacia un modelo de gestión estratégica”, realizado en la Universidad Estatal a Distancia de Costa Rica (tesis doctoral). Universidad Estatal a Distancia. Costa Rica.

Jiménez de La Jara, M. (2008). ¿Cómo medir la percepción de la responsabilidad social en los diversos estamentos de la universidad? Una experiencia concreta. Revista Educación Superior y Sociedad, 13(2), Editorial Nueva Época, Unesco, 139. Recuperado de https://unesdoc.unesco.org/ark:/48223/pfooo0182067

Martín, V. (2012). Universidad socialmente responsable: Universidad éticamente inteligente. Megatendencias sobre la vida, lo humano y el futuro. Opción: Revista de Ciencias Humanas y Sociales, 28(68), 233-240. Recuperado de https:// dialnet.unirioja.es/servlet/articulo? codigo $=4226318$

Vallaeys, F.; De la Cruz, C. y Sasia, P. (2009), Responsabilidad Social Universitaria. Manual de primeros pasos. México: Banco Interamericano de Desarrollo, McGraw-Hill. Recuperado de http://idbdocs.iadb.org/wsdocs/getdocument. aspx?docnum $=35125786$ 